\title{
BO Pegasi: A candidate on the breaking stage of the TRO cycle
}

\author{
Qian Shengbang*
}

\author{
Yunnan Observatory, Chinese Academy of Sciences, PO Box 110, 650011 Kunming, PR China \\ National Astronomical Observatories, Chinese Academy of Science (NAOCAS) \\ United Laboratory of Optical Astronomy, Chinese Academy of Science (ULOAC)
}

Received 5 June 2001 / Accepted 28 January 2002

\begin{abstract}
A detailed period study of the short-period $(P=0.58)$ close binary system, BO Peg, is presented based on the analysis of its $\mathrm{O}-\mathrm{C}$ curve. It is found that the orbital period shows a continuous decrease with a rate $\mathrm{d} P / \mathrm{d} t=-1.26 \times 10^{-7}$ days/year while it undergoes a possible small-amplitude oscillation. If the period oscillation is cyclic, it can be explained either by the light-time effect via the presence of an unseen third body or by the magnetic activity cycle of the secondary component. The continuous period decrease suggests a secular mass transfer from the more massive to the less massive component, which is in agreement with the near-contact configuration with a lobe-filling primary (Yamasaki \& Okazaki 1986). These observations, as seen in RT Scl, FT Lup, TT Her, RS Ind, and V388 Cyg, are consistent with the Thermal Relaxation Oscillation (TRO) theory in that the primary component is transferring mass to the secondary in the broken-contact phase. To satisfy such a decrease in the orbital period, a conservative mass transfer rate (with no magnetic effect) would be of the order of $\mathrm{d} M / \mathrm{d} t=-1.53 \times 10^{-7} M_{\odot} /$ year.
\end{abstract}

Key words. stars: binaries: close - stars: individuals: BO Peg - stars: evolution

\section{Introduction}

The light variability of the eclipsing binary system, BO Pegasi, was discovered by Hoffmeister (1935). Later, Jensch (1935) presented a photographic light curve and determined the orbital period to be 0.58044 days. According to the 4th edition of the General Catalogue of Variable Stars (GCVS), its spectral type is A4. The first photoelectric light curves in $B$ and $V$ were published by Yamasaki \& Okazaki (1986). As for other close binary systems, such as TT Her, FT Lup, RT Scl, RS Ind, and V388 Cyg, the light curve is a typical EB-type showing a continuous light variation, but with unequal depth in the minima. A photometric solution by Yamasaki \& Okazaki (1986) indicated that BO Peg is a near-contact binary system. The primary component fills the critical Roche lobe, while the secondary is detached. Here, the period change of this eclipsing binary systems is studied, and then the structure and evolutionary state of the system are discussed.

\section{O-C curve analysis}

Epochs and orbital periods have been given by Jensch (1935), Whitney (1957), and Yamasaki \& Okazaki (1986). Yamasaki \& Okazaki (1986) derived a linear ephemeris

\footnotetext{
* e-mail: qsb@netease.com
}

and suggested that period changes of the system occurred around HJD 2434000 and HJD 2446000. 28 times of light minimum were given by Yamasaki \& Okazaki (1986) and 24 timings were compiled in the Eclipsing Binaries Minima Database (EBMD) (available at http://www.oa.uj.edu.pl/ktt/krttk_dn.html). Recently, three photoelectric timings were published by Agerer \& Hubscher $(1996,1998)$ and by Agerer et al. (2001), and one CCD time of minimum light was given by Nelson (2001). The $\mathrm{O}-\mathrm{C}$ values of all those times of light minimum are calculated with the linear ephemeris given in the GCVS:

Min. $I=2445612.3230+0.5804301 \times E$.

The corresponding $\mathrm{O}-\mathrm{C}$ diagram is shown in Fig. 1.

As seen in Fig. 1, the period of BO Peg is variable and complex. The general trend of the $\mathrm{O}-\mathrm{C}$ curve can be described by a downward parabolic curve indicating a secular period decrease. The photoelectric light curves of Yamasaki \& Okazaki (1986) showed that the primary minimum of BO Peg is rather sharp and deep (up to 0.8 ) which make it a suitable object for times of light minimum. Thus we expect that the visual or photographic data should be reliable within the error of \pm 0 d 01 . With weights 8 for photoelectric or CCD observations and 1 for photographic or visual data, the following quadratic 


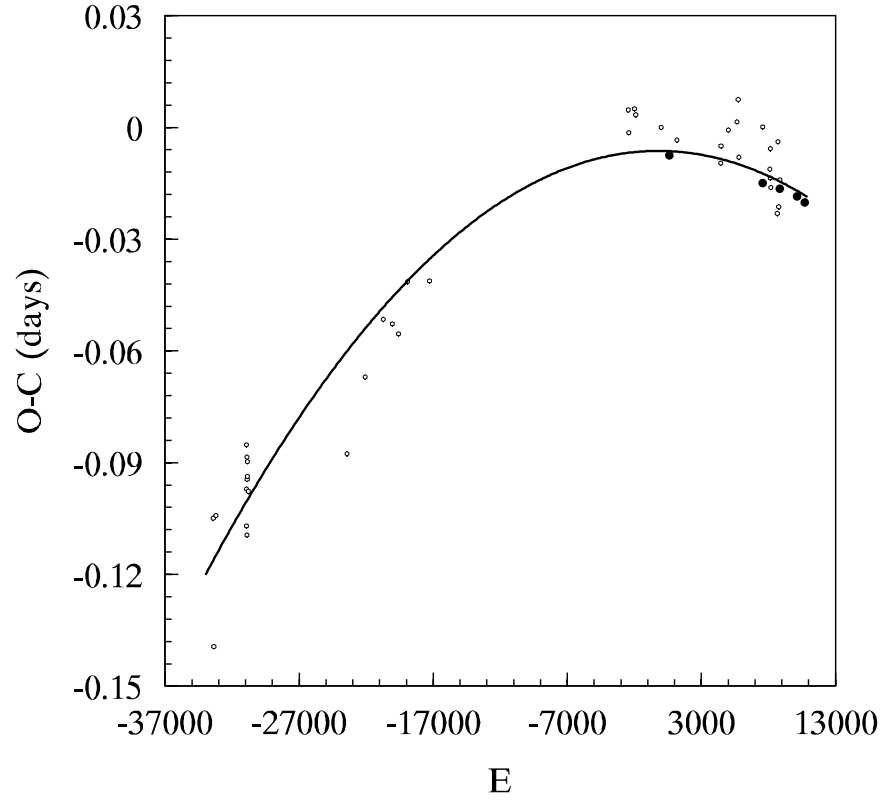

Fig. 1. O-C curve of BO Peg, showing the quadratic fit to the data. Circles represent visual or photographic observations and dots represent photoelectric or CCD data.

ephemeris:

$$
\begin{aligned}
\text { Min. } I= & 2445612.3167(6)+0.58043006(2) \\
& \times E-9.98(7) \times 10^{-11} \times E^{2},
\end{aligned}
$$

is obtained. From the quadratic term of this ephemeris, a continuous period decrease with rate of $\mathrm{d} P / \mathrm{d} t=-1.26 \times$ $10^{-7}$ days/year is determined. The typical deviations on the epochs of timings in Fig. 1, especially for photographic or visual data, are large (up to $0.015 \mathrm{~d}$ ). Therefore, the fit only represents a mean trend which is nevertheless consistent with the possibility of a cyclic period change.

The residuals $\left((\mathrm{O}-\mathrm{C})^{\prime}\right.$ values $)$ based on the quadratic ephemeris are plotted in Fig. 2, omitting two values which show large deviations from the general trend. As seen in the figure, a periodic oscillation may exist. With the same weights as those used in the previous paragraph, a weighted least-squares solution leads to the cyclic ephemeris:

$$
\begin{aligned}
(\mathrm{O}-\mathrm{C})^{\prime}= & -0.0012( \pm 0.0013)+0.0054( \pm 0.0018) \\
& \times \sin \left(0.0090^{\circ} E+83.7^{\circ}\left( \pm 22.0^{\circ}\right)\right) .
\end{aligned}
$$

This yields a periodic change with an amplitude of $A=0$ d.0054 and a period of $P_{3}=63.6$ years. The cyclic variation of the $(\mathrm{O}-\mathrm{C})^{\prime}$ curve indicates that the period change may be cyclic. Using the following equation:

$\Delta P=\sqrt{2\left[1-\cos \left(2 \pi P / P_{3}\right)\right]} \times A$,

the amplitude of the period variation can be calculated to be: $\Delta P=0.84 \times 10^{-6}$ days. However, since the $(\mathrm{O}-\mathrm{C})^{\prime}$ values show large scatter and the periodic variation is mainly formed by the visual and photographic observations, more

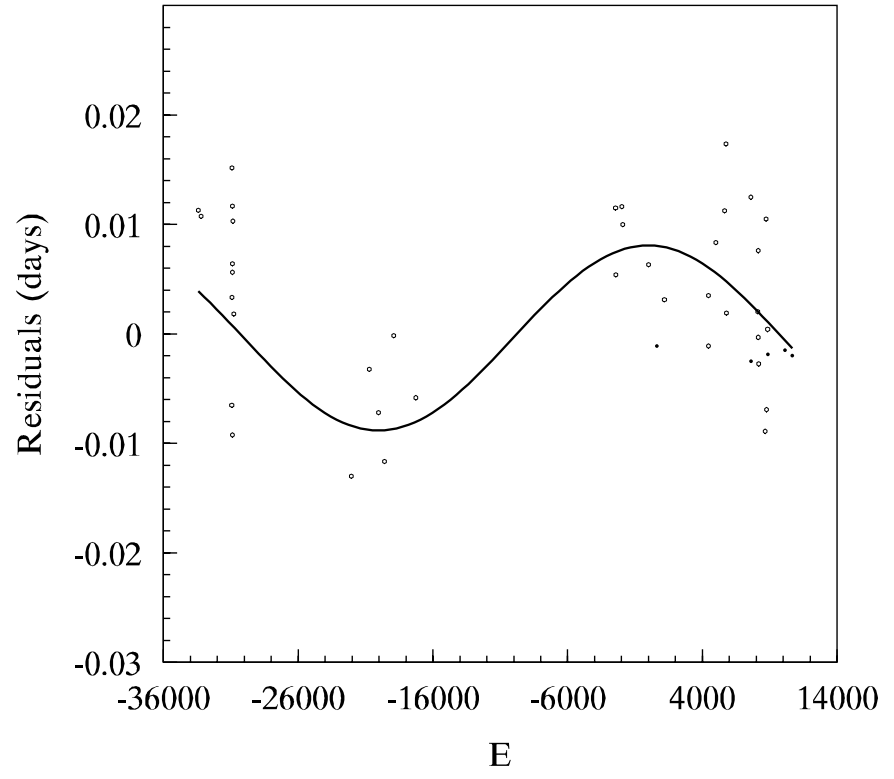

Fig. 2. Residuals of BO Peg based on the quadratic ephemeris. Symbols are as in Fig. 1.

photoelectric or CCD data are needed to verify this finding. Moreover, the residuals used here only cover one cycle and must be extended before this issue can be resolved.

\section{Mechanisms for orbital period variations}

\subsection{Periodic mechanisms}

Possible periodic mechanisms include a) the light-time effect via the existence of a third body and b) the magnetic activity cycle in the secondary.

a) For the light-time effect, I calculate the value $a_{12}^{\prime} \sin i^{\prime}$. Then using:

$f(m)=\frac{\left(M_{3} \sin i^{\prime}\right)^{3}}{\left(M_{1}+M_{2}+M_{3}\right)^{2}}=\frac{4 \pi^{2}}{G P_{3}^{2}} \times\left(a_{12}^{\prime} \sin i^{\prime}\right)^{3}$,

we can obtain a small mass function $f(m)=0.0002 M_{\odot}$ for the additional body. Taking the absolute parameters given by Yamasaki \& Okazaki (1986), $M_{1}=1.9 M_{\odot}$ and $M_{2}=1.0 M_{\odot}$, the values of the masses and the orbital radii of the third body for several different values of $i^{\prime}$ are computed and listed in Table 1 . If we assume that the third body is coplanar to the orbit of the eclipsing pair (i.e., $\left.i^{\prime}=79.1^{\circ}\right)$, the value of the mass and the orbital radius of the additional body is computed to be $m_{3}=0.13 M_{\odot}$ and $a_{3}=22.2 \mathrm{AU}$ respectively. In this case, the third body would be very difficult to detect because its luminosity is extremely low.

b) BO Peg contains an A7IV-V-type primary and an early G-type secondary (Allen 1973; Yamasaki \& Okazaki 1986). The oscillation in the orbital period can also be explained as a consequence of a possible magnetic cycle in this secondary component. The model was first proposed by Matese \& Whitmire (1983) and Applegate (1992) who proposed that a quasi-periodic exchange of angular 
Table 1. The values of the masses and the orbital radii of the possible third body in BO Peg.

\begin{tabular}{lll}
\hline Parameters & Values & Units \\
\hline \hline$A$ & 0.0054 & days \\
$P_{3}$ & 63.6 & years \\
$a_{12}^{\prime} \sin i^{\prime}$ & 0.94 & $\mathrm{AU}$ \\
$f(m)$ & $2.0 \times 10^{-4}$ & $M_{\odot}$ \\
$m_{3}\left(i^{\prime}=90^{\circ}\right)$ & 0.12 & $M_{\odot}$ \\
$m_{3}\left(i^{\prime}=70^{\circ}\right)$ & 0.13 & $M_{\odot}$ \\
$m_{3}\left(i^{\prime}=50^{\circ}\right)$ & 0.16 & $M_{\odot}$ \\
$m_{3}\left(i^{\prime}=30^{\circ}\right)$ & 0.25 & $M_{\odot}$ \\
$m_{3}\left(i^{\prime}=10^{\circ}\right)$ & 0.81 & $M_{\odot}$ \\
$a_{3}\left(i^{\prime}=90^{\circ}\right)$ & 22.7 & $\mathrm{AU}$ \\
$a_{3}\left(i^{\prime}=70^{\circ}\right)$ & 22.1 & $\mathrm{AU}$ \\
$a_{3}\left(i^{\prime}=50^{\circ}\right)$ & 22.0 & $\mathrm{AU}$ \\
$a_{3}\left(i^{\prime}=30^{\circ}\right)$ & 21.8 & $\mathrm{AU}$ \\
$a_{3}\left(i^{\prime}=10^{\circ}\right)$ & 19.4 & $\mathrm{AU}$ \\
\hline \hline
\end{tabular}

momentum between the inner and the outer parts in the convection zone may induce a modulation of its quadruple moment and therefore its orbital period. Recently, the details of Applegate's mechanism were studied by Lanza et al. (1998). They pointed out that, in addition to the redistribution of the internal angular velocity, the change in the azimuthal field intensity can likewise produce a change in the oblateness of the active component. The stability of the azimuthal magnitude field was discussed by Lanza \& Rodonò (1999) by considering a more general magnetic field geometry. If the period oscillation of the system results from the magnetic activity cycle in the secondary, then with the physical parameters $M_{2}=1.0 M_{\odot}$, $R_{2}=1.3 R_{\odot}$, and $a=4.2 R_{\odot}$ (Yamasaki \& Okazaki 1986), and using:

$\frac{\Delta P}{P}=-9\left(\frac{R_{2}}{a}\right)^{2} \frac{\Delta Q}{M_{2} R_{2}^{2}}$,

the required variation of the quadruple moment $\Delta Q$ in order to reproduce period change $\Delta P=0.84 \times 10^{-6}$ is $\Delta Q=2.73 \times 10^{49} \mathrm{~g} \mathrm{~cm}^{2}$.

\subsection{Non-periodic mechanisms}

The secular decrease component in the orbital period of BO Peg can be interpreted as being caused by either mass transfer from the more massive to the less massive component or by angular momentum loss (AML). If such an orbital period decrease is due to a conservative mass transfer (with no magnetic effect), then using:

$\Delta P / P=3\left(M_{1} / M_{2}-1\right) \Delta M_{1} / M_{1}$,

the mass transfer rate of $\mathrm{d} M / \mathrm{d} t=-1.53 \times 10^{-7} M_{\odot} /$ year is found. However, the secular period decrease may be caused by AML via magnetic stellar wind. A general equation for AML described in detail by Vilhu (1992) is:

$\mathrm{d} J / \mathrm{d} t=-1.0 \times 10^{42} K^{2} f^{-2} M R^{\gamma}(P / 3)^{-\alpha}$

where $M$ and $R$ are the mass and the radius of the component, and $P$ is the orbital period. Given Vilhu's (1982) parameters, $K^{2}=0.1, f=1, \gamma=4$, and $\alpha=1.5$, the AML rates computed with Eq. (8) are $-2.35 \times 10^{43}$ and $-2.68 \times 10^{42} \mathrm{~g} \mathrm{~cm}^{2} \mathrm{esc}^{-1}$ year $^{-1}$ which correspond to orbital period decrease rates of $-1.87 \times 10^{-9}$ and $-2.68 \times 10^{-10}$ days/year for the two components. The computed rates are two orders smaller than the observed value. Using Guinan \& Bradstreet's (1988) equation:

$$
\begin{aligned}
\mathrm{d} P / \mathrm{d} t= & -1.1 \times 10^{-8} q^{-1}(1+q)^{2} \\
& \times\left(M_{1}+M_{2}\right)^{-5 / 3} k^{2}\left(M_{1} R_{1}^{4}+M_{2} R_{2}^{4}\right) P^{-7 / 3}
\end{aligned}
$$

and taking the value $k^{2}=0.1$, we can obtain $\mathrm{d} P / \mathrm{d} t=$ $-6.62 \times 10^{-8}$ days/year which is also smaller than the observed rate. These results may demonstrate that a magnetic braking mechanism is not sufficient to explain the secular period decrease.

\section{Discussion and conclusions}

The secular decrease component in the orbital period may not be caused by magnetic braking, since the calculated $\mathrm{d} P / \mathrm{d} t$ is much smaller than that observed. The continuous decrease, interpreted as primary-to-secondary conservative mass transfer, implies that $\mathrm{d} M / \mathrm{d} t=-1.53 \times$ $10^{-7} M_{\odot} /$ year. Yamasaki \& Okazaki (1986) showed that the primary to be filling its critical Roche lobe, in agreement with the period decrease. These findings suggest that BO Peg is evolving into contact, as is thought to be occurring in FT Lup, RT Scl, RS Ind, V388 Cyg, and TT Her. These are all near-contact systems with lobe-filling primaries and secular period decreases. Table 2 gives the rate of period change, the timescale for period change, and the corresponding mass transfer rate.

Several theories of the structure and evolution of $\mathrm{W}$ UMa type overcontact binaries were proposed in the 1970s. Assuming conservation of mass and angular momentum, Thermal Relaxation Oscillation (TRO) models (Lucy 1976; Flannery 1976; Robertson \& Eggleton 1977) predicted oscillation between semi-detached and slightly overcontact configurations. In the contact stage, the direction of mass transfer is opposite to that of the energy transfer and causes a rapid increase in separation. The system then reaches a configuration with the primary filling its lobe and transferring material to the secondary. Recently, orbital period changes of 27 overcontact binaries $\left(M_{1} \geq 1.35 M_{\odot}\right)$ were checked by Qian (2001). Apart from AW UMa, with the most extreme mass ratio among W UMa stars, none showed a period decrease and 21 had increasing $P$. The high temperatures of these stars suggest a decreasing efficiency of the angular momentum loss by magnetic braking. The period increases are consistent with the TRO theories. For the semi-detached systems of Table 2, the observations suggest the system is in the broken contact stage of TRO with evolution towards thermal contact.

Acknowledgements. This work is partly supported by Chinese Natural Science Foundation and National Key Fundamental Research Project through grant G1999075405. The author 
Table 2. Orbital period decrease rates of several near-contact binaries.

\begin{tabular}{lllllll}
\hline Star name & Sp. & $P$ (days) & $\mathrm{d} P / \mathrm{d} t$ (days/year) & $P / \mathrm{d} P / \mathrm{d} t$ (years) & $\mathrm{d} M / \mathrm{d} t\left(M_{\odot} /\right.$ year $)$ & Ref. \\
\hline \hline FT Lup & F2V+V5-7 & 0.4700831 & $-3.54 \times 10^{-7}$ & $1.33 \times 10^{6}$ & $1.3 \times 10^{-7}$ & $(1)$ \\
RT Scl & F0V & 0.51156012 & $-2.58 \times 10^{-7}$ & $1.96 \times 10^{6}$ & $1.4 \times 10^{-8}$ & $(2)(3)$ \\
BO Peg & A7IV-V+G & 0.58043288 & $-1.26 \times 10^{-7}$ & $4.60 \times 10^{6}$ & $1.5 \times 10^{-7}$ & $(4)$ \\
RS Ind & F4+G5 & 0.62405225 & $-4.14 \times 10^{-7}$ & $1.76 \times 10^{6}$ & - & $(5)$ \\
V388 Cyg & A3 & 0.8590515 & $-4.33 \times 10^{-7}$ & $1.98 \times 10^{6}$ & $1.9 \times 10^{-6}$ & $(6)$ \\
TT Her & F2V & 0.91208023 & $-3.53 \times 10^{-7}$ & $2.58 \times 10^{6}$ & $1.0 \times 10^{-7}$ & $(7)$ \\
\hline \hline
\end{tabular}

References in Table 2:

(1) Lipari \& Sistero (1986); (2) Hilditch \& King (1986); (3) Duerbeck \& Karimie (1978); (4) Present; (5) Marton et al. (1990);

(6) Milano \& Russo (1983); (7) Milano et al. (1989).

thanks the anonymous referee for his/her useful suggestions and comments.

\section{References}

Agerer, F., \& Hubscher, J. 1996, IBVS, 4832

Agerer, F., \& Hubscher, J. 1998, IBVS, 4606

Agerer, F., Dahm, M, \& Hubscher J. 2001, IBVS, 5017

Allen, C. W. 1973, Astrophysical Quantities (3d ed.; London: Athlone Press), 209

Applegate, J. H. 1992, APJ, 385, 621.

Duerbeck, H. W., \& Karimie, M. T. 1978, IBVS, 1617

Guinan, E. F., \& Bradstreet, D. H. 1988, in Formation and

Evolution of Low Mass Star, A. K. Dupree, M. T. V. T. Lago eds (Kluwer Acad. Publ.), 345

Flannery, B. P. 1976, APJ, 205, 317

Hilditch, R. W., \& King, D. J. 1986, MNRAS, 223, 581

Hoffmeister, C. 1935, Astr. Nach, 255, 401

Jensch, A. 1935, Astr. Nach., 255, 417
Lanza, A. F., \& Rodonò, M. 1999, A\&A, 349, 887

Lanza, A. F., Rodonò, M., \& Rosner, R. 1998, MNRAS, 296, 893

Lipari, S. L., \& Sistero, R. F. 1986, MNRAS, 220, 883

Lucy, L. B. 1976, APJ, 205, 208

Marton, S. F., \& Grio, G. 1983, MNRAS, 203, 235

Matese, S. F., Grieco, A., Lapasset, E., et al. 1990, A\&A, 237, 73

Matese, J. J., \& Whitmire, D. P. 1983, A\&A, 117, L7

Milano, L., \& Russo, G. 1983, MNRAS, 203, 235

Milano, L., Barone, F., Mancuso, S., Russo, G., \& Vittone, A. A., 1989, A\&A, 210, 181

Nelson, R. H. 2001, IBVS, 5040

Qian, S. B. 2001, MNRAS, 328, 914

Robertson, J. A., \& Eggleton, P. P. 1977, MNRAS, 179, 359

Vilhu, O. 1982, A\&A 109, 17

Vilhu, O. 1991, IAU Symp., 151, 61

Yamasaki, A., \& Okazaki, A. 1986, PASP, 98, 1325 